\title{
Quellen und Literatur
}

\section{Periodika}

Gewerkschaftliche Monatshefte, hrsg. vom Bundesvorstand des DGB, Jahrgänge 1950-1998.

Gewerkschaftszeitung, Monatszeitschrift des ADGB, Jahrgänge 1929-1933.

textil-bekleidung, Mitgliederzeitung der Gewerkschaft Textil-Bekleidung, Jahrgänge 1949-1997.

\section{Protokolle, Geschäftsberichte und Archivalien}

Außenhandelsvereinigung des Deutschen Einzelhandels e. V.: Jahresbericht 1974/75.

Bauer, Walter: Schreiben an Werner Bock vom 10. Juli 1962, AdsD 5/GTBA410250.

BBI: Protokoll der Mitgliederversammlung vom 10. Mai 1988, BWA V11 10.

BBI: Protokolle der Mitgliederversammlungen 1948-1968, BWA.

BDA: Protokoll der Sitzung des Arbeitskreises Frauenlohn vom 27. März 1953, Bayerisches Wirtschaftsarchiv, BWA No2 61.

DGB: Geschäftsberichte des Bundesvorstandes, 1949-1997, Zentralbibliothek der IG Metall.

DGB: Protokoll der 3. Bundesfrauenkonferenz, 25.-27. Mai 1959 in Bremen, Zentralbibliothek der IG Metall.

DGB: Protokoll des Gründungskongresses, 12.-14. Oktober 1949 in München, Zentralbibliothek der IG Metall.

DGB: Protokolle der Bundeskongresse, 1949-1998, Zentralbibliothek der IG Metall.

DGB: Protokoll der Sitzung des Bundesvorstandes vom 7.3.1961, AdsD 5/ DGBA1000533.

Gesamttextil: Beschluss des Präsidiums vom 3. Juni 1948, in: Protokoll der Präsidiumssitzung vom 3. Juni 1948, Bayerisches Wirtschaftsarchiv, BWA No2 51.

Gesamttextil: Protokoll der Beiratssitzung vom 13. Dezember 1953, BWA No2 55.

Gesamttextil: Protokoll zur Gründung des Sozialpolitischen Ausschusses, 23./24. Juli 1948, BWA No2 53. 
Gesamttextil: Protokolle der Hauptausschusssitzungen, 1948-1967, BWA.

Gesamttextil: Protokolle der Präsidiumssitzungen, 1948-1953, BWA.

Gesamttextil: Protokolle der Sitzungen des Sozialpolitischen Ausschusses, 19481958, BWA.

Gesamttextil: Protokolle der Sitzungen des Arbeitgeberkreises, 1959-1967, BWA.

Gesamttextil: Rundschreiben der Sozialpolitischen Abteilung vom 26. Juni 1952, BWA No2 58.

Gesamttextil: Vertrauliches Rundschreiben vom 20. November 1952, BWA No2 60. GTB: Aktennotiz über ein Gespräch von Karl Buschmann mit Dr. Töpfer, dem Textilreferenten des BMWi, am 12. November 1958, AdsD 5/GTBA100140.

GTB: Bericht der Verwaltungsstelle Jöllenbeck andatiert (1962), AdsD 5/ GTBA410212.

GTB: Geschäftsberichte der Abteilung Frauen zu den Frauenkonferenzen, 19571971 (Privatbesitz Donath).

GTB: Geschäftsberichte des Hauptvorstandes, 1949-1997 (Privatbesitz Donath).

GTB: informationen für Vertrauensleute, 1978 (Privatbesitz Donath).

GTB, Niederschrift der Beiratssitzung vom 10./11.2.1955, AdsD 5/GTBA030001.

GTB: Presseerklärung vom 22. Februar 1965, AdsD 5/GTBA410294.

GTB: Pressenachrichten vom 30. April 1965, AdsD 5/GTBA410294.

GTB: Protokolle der Beiratssitzungen, 1949-1997, AdsD.

GTB: Protokolle der Sitzungen des Geschäftsführenden Hauptvorstandes, 19491998, AdsD.

GTB: Protokolle der Gewerkschaftstage, 1949-1997 (Privatbesitz Donath).

GTB: Protokolle der Zentralen Frauenkonferenzen, 1957-1971, Zentralbibliothek der IG Metall.

GTB: Tarifakten 1965, AdsD 5/GTBA410294.

IG Metall: Detaillierte Auswertung der Betriebsratswahlen 1990 (unveröffentlicht).

IG Metall: Ergebnisse der Betriebsratswahl 2002 (unveröffentlicht).

IG Metall: Geschäftsbericht 1971-1973, Zentralbibliothek der IG Metall.

IG Metall: Interne Integrationsrichtlinie (unveröffentlicht).

IG Metall: Wortprotokoll des 4. Außerordentlichen Gewerkschaftstages, 11. Oktober 1997 in Mannheim, Zentralbibliothek der IG Metall.

Keller, Berthold: Handschriftliche Notizen von der Sitzung des GHV mit den Bezirksleitern am 6./7. Mai 1965, AdsD 5/GTBA410294.

Rationalisierungsschutz-Tarifverträge für die Textil- und Bekleidungsindustrie, 1988, IG-Metall-Tarifarchiv.

Stiftung Bildung, Erholung, Gesundheit (vormals »Stiftung zur Förderung von Bildung und Erholung der Arbeitnehmer in der Miederindustrie«): Geschäftsberichte 1965-2017, Bibliothek der Kritischen Akademie, Inzell.

Stiftung zur Förderung von Bildung und Erholung der Arbeitnehmer in der Miederindustrie $\rightarrow$ siehe »Stiftung Bildung, Erholung, Gesundheit«. 
Trost, Paul (1965): Handschriftliche Aktennotiz vom 26.7.1965, AdsD 5/GTBA410254. Verband der Textilindustrie Westfalen: Schreiben des Vorstandes an den Arbeitsminister des Landes Nordrhein-Westfalen, 11. März 1953, BWA No2 61.

Westfälischer Rationalisierungstarifvertrag, abgeschlossen zwischen dem Verband der Textilindustrie Westfalen, Münster, und der Gewerkschaft TextilBekleidung, Düsseldorf, 13. Februar 1964, AdsD 5/GTBA410255.

\section{Literatur}

Abelshauser, Werner (2004): Deutsche Wirtschaftsgeschichte seit 1945, München: C. H. Beck.

Anonym (o. J.): Die Genossen unter sich. Auf Anregung der Rheinischen Gruppe des Vereins zur Förderung staats- und gesellschaftspolitischer Bildung e. V. (Privatbesitz Donath).

Angster, Julia (2003): Konsenskapitalismus und Sozialdemokratie. Die Westernisierung von SPD und DGB, München: Oldenbourg.

Arens, Willi (1990): GTB-Programm, in: textil-bekleidung, Ausgabe 11/1990, S. 3.

Arens, Willi (1994): 1994 - Ein Ausblick, in: textil-bekleidung, Ausgabe 1/1994, S. 3.

Armingeon, Klaus (1988): Die Entwicklung der westdeutschen Gewerkschaften. 1950-1985, Frankfurt am Main/New York: Campus.

Aulenbacher, Brigitte/Goldmann, Monika (Hrsg) (1993): Transformationen im Geschlechterverhältnis, Frankfurt am Main/New York: Campus.

Barthes, Roland (1983): Die Sprache der Mode, übersetzt von Horst Brühmann, 3. Aufl., Frankfurt am Main: Suhrkamp (französischer Originaltitel: »Système de la mode $\ll)$.

Bastian, Renate (1979): Am Scheideweg. Zum Programm der Gewerkschaft TextilBekleidung, in: nachrichten, Nachrichten-Reihe Nr. 14.

BDA = Bundesvereinigung der Deutschen Arbeitgeberverbände (Hrsg.) (1965): Der Arbeitgeber, Sonderausgabe vom 20.3.1965.

$\mathrm{BDA}=$ Bundesvereinigung der Deutschen Arbeitgeberverbände (1978): Katalog zur Koordinierung der lohn- und tarifpolitischen Fragen, in: Frankfurter Rundschau, Ausgabe vom 26.1.1979.

Beyer, Andrea (1991): Tarifpolitik in strukturschwachen Branchen. Eine empirische Untersuchung der Druckerei- und der Textilindustrie in der Bundesrepublik Deutschland, München: Minerva.

Bierbaum, Frank (1992): Strategisches Verhalten in stagnierenden Branchen. Eine Darstellung am Beispiel der deutschen Textilindustrie, St. Gallen (Dissertation).

Bispinck, Reinhard/Bauer, Götz/Föhr, Merle/Schmidt, Ulrich/Taube, Andrea/ Wollensack, Monika/Ziouziou, Jasmina (2019): 70 Jahre Tarifvertragsgesetz. 
Stationen der Tarifpolitik von 1949 bis 2019, Elemente qualitativer Tarifpolitik Nr. 85, Düsseldorf: Wirtschafts und Sozialwissenschaftliches Institut (WSI) der Hans-Böckler-Stiftung.

Bispinck, Reinhard/Schulten, Thorsten/Peeter Raane (Hrsg.) (2008): Wirtschaftsdemokratie und expansive Lohnpolitik. Zur Aktualität von Viktor Agartz, Hamburg: VSA-Verlag.

Bohnsack, Anke (1985): Nicht nur von anderen fordern ... Wie sieht es in Parteien und Gewerkschaften aus?, in: Däubler-Gmelin, Herta/Pfarr, Heide M./Weg, Marianne (Hrsg.): »Mehr als nur gleicher Lohn!«. Handbuch zur beruflichen Förderung von Frauen, Hamburg, VSA-Verlag, S. 66-70.

Borchardt, Bernd u. a. (2014): Crimmitschau. Geschichte einer Stadt, Band 2, Horb am Neckar: Geiger-Verlag.

Breit, Ernst (1984): Alle Möglichkeiten nutzen, in: textil-bekleidung, Ausgabe $1 / 1984$, S. 3 .

Brenner, Otto (1971): Was bedeutet die Reform des DGB?, in: Gewerkschaftliche Monatshefte, Ausgabe 4/1971, S. 209-212.

Brücher, Bodo/Buschmann, Inge/Link, Bernd (2004): Starke Frauen, Arbeitskampf und Solidarität. Die Gewerkschaft Textil-Bekleidung in der Region Bielefeld 1849-1998, hrsg. von Hilde Junker-Seeliger und IG Metall Bielefeld, Bielefeld: Hans Gieselmann Druck und Medienhaus.

Bundesagentur für Arbeit (2021): Tabellen - Arbeitslosigkeit im Zeitverlauf: Entwicklung der Arbeitslosenquote (Strukturmerkmale). Deutschland, West, Ost, https://statistik.arbeitsagentur.de/Statistikdaten/Detail/Aktuell/iiia4/alozeitreihe-dwo/alo-zeitreihe-dwo-b-o-xlsx.xlsx (Abruf am 17.2.2021).

Buschmann, Karl (1953): Lohn- und Tarifpolitik in Gegenwart und Zukunft, Rede auf dem 3. Ordentlicher Gewerkschaftstag der GTB, in: Protokoll des 3. Ordentlichen Gewerkschaftstages, 16.-19. Juni 1953 in Düsseldorf, S. 136-152.

Buschmann, Karl (1957): Die technische Entwicklung in der Textilwirtschaft und ihre Auswirkungen auf die Arbeitnehmerschaft, Rede auf dem 5. Ordentlichen Gewerkschaftskongress der GTB, in: Protokoll des 5. Ordentlichen Gewerkschaftskongresses, 2.-5. Juli 1957 in Kassel, S. 88-106.

Buschmann, Karl (1971): Wir brauchen einen starken Gewerkschaftsbund, in: Gewerkschaftliche Monatshefte, Ausgabe 4/1971, S. 206-209.

Däubler-Gmelin, Herta (1977): Frauenarbeitslosigkeit - oder Reserve zurück an den Herd, Reinbek bei Hamburg: Rowohlt.

Deppe, Frank/Fülberth, Georg/Harrer, Jürgen (Hrsg.) (1977): Geschichte der deutschen Gewerkschaftsbewegung, Köln: Pahl-Rugenstein.

Der Spiegel (1954): Mindestens das Doppelte, in: Ausgabe 33 vom 11.8.1954, S. 5-8, www.spiegel.de/spiegel/print/d-28957158.html (Abruf am 24.2.2021).

Der Spiegel (1959): Ostkontakte - Anruf vom Politruk, in: Ausgabe 15 vom 8.4.1959, S. 26-28, www.spiegel.de/spiegel/print/d-42625028.html (Abruf am 8.2.2021). 
Der Spiegel (1969): Arbeitskampf - Wilde Woche , in: Ausgabe 38 vom 15.9.1969,

S. 30-32, www.spiegel.de/spiegel/print/d-45547764.html (Abruf am 25.2.2021).

Der Spiegel (1981): SPD - Etwas dünn, in: Ausgabe 50 vom 7.12.1981, S. 21-23, www. spiegel.de/spiegel/print/d-14351365.html (Abruf am 15.2.2021).

Der Spiegel (1982): Gut getarnt im Dickicht der Firmen. Neue Heimat: Die dunklen Geschäfte von Vietor und Genossen, in: Ausgabe 6 vom 8.2.1982, S. 92-104, www.spiegel.de/spiegel/print/d-14342289.html (Abruf am 15.2.2021).

Der Spiegel (1984): 35-Stunden-Woche - Zufällig über den Weg, in: Ausgabe 13 vom 26.3.1984, S. 19-21, www.spiegel.de/spiegel/print/d-13509999.html (Abruf am 13.2.2021).

Der Spiegel (1984): Arbeitszeit - Noch viel mobilisieren, in: Ausgabe 3 vom 16.1.1984, S. 81-83, www.spiegel.de/spiegel/print/d-13509226.html (Abruf am 13.2.2021).

Der Spiegel (1984): Rente - Fein gemacht, in: Ausgabe 11 vom 12.3.1984, S. 28 f., www.spiegel.de/spiegel/print/d-13508460.html (Abruf am 13.2.2021).

Der Spiegel (1988): Gewerkschaften - Stellung unterlaufen, in: Ausgabe 32 vom 8.8.1988, S. 79-82, www.spiegel.de/spiegel/print/d-13529551.html (Abruf am 15.2.2021).

Deutsche Rentenversicherung (2009): Heiratserstattung, http://forschung.deut sche-rentenversicherung.de/FdzPortalWeb/FdzFaqDispDetail.do?id=84 (Abruf am 11.2.2021).

Deutscher Bundestag (2018): Vor 100 Jahren: Reichswahlgesetz führt das Frauenwahlrecht ein, www.bundestag.de/dokumente/textarchiv/2018/kw48-kalen derblatt-frauenwahlrecht-580156 (Abruf am 18.1.2021).

Die Zeit (1980): IG Chemie - Das böse Wort vom Ausmauscheln, in: Ausgabe 7 vom 8.2.1980, www.zeit.de/1980/07/das-boese-wort-vom-ausmauscheln (Abrufam 15.2.2021).

DGB = Deutscher Gewerkschaftsbund (o. J.): Der Weg zum ersten »Bündnis für Arbeit«, www.dgb.de/themen/++co++62243886-1902-11df-6dd1-00093d1ofae2 (Abruf am 17.2.2021).

DGB = Deutscher Gewerkschaftsbund (Hrsg.) (1949): Die Gewerkschaftsbewegung in der britischen Zone, Köln: Bund-Verlag.

Dobbert, Alfred (1966): Erlebnisse in den zwanziger Jahren, in: textil-bekleidung. Mitgliederzeitung der Gewerkschaft Textil-Bekleidung, Ausgabe 10/1966, S. $16 \mathrm{f}$.

DTAV = Hauptvorstand des Deutschen Textilarbeiter-Verbands (1928): Crimmitschau 1902-1928. Blätter der Erinnerung an Sachsens bedeutsamsten Arbeitskampf, Berlin (Nachdruck 1991, Düsseldorf: WI-Verlag).

Esser, Josef (1982): Gewerkschaften in der Krise. Die Anpassung der deutschen Gewerkschaften an neue Weltmarktbedingungen, Frankfurt am Main: Suhrkamp. 
Esser, Josef (2003): Funktion und Funktionswandel der Gewerkschaften in Deutschland, in: Schroeder, Wolfgang/Weßels, Bernhard: Die Gewerkschaften in Politik und Gesellschaft der Bundesrepublik Deutschland, Wiesbaden: Westdeutscher Verlag, S. 65-85.

Erhard, Ludwig (1958): Es liegt jetzt an uns, ob wir bestehen werden, in: Die Zeit, Ausgabe 8 vom 20.2.1958, www.zeit.de/1958/08/es-liegt-jetzt-an-uns-ob-wirbestehen-werden (Abruf am 26.1.2021).

Fehmel, Thilo (2010): Konflikte um den Konfliktrahmen. Die Steuerung der Tarifautonomie, Wiesbaden: VS Verlag für Sozialwissenschaften.

Frankfurter Allgemeine Zeitung (1965): Der Kleinkrieg der IG Textil, in: Ausgabe vom 15.3.1965.

Frankfurter Neue Presse (1959): DGB-Funktionär setzt sich ab, in: Ausgabe vom 23.5.1959.

Frick, Lothar (Hrsg.) (2015): »Heraus zum Massenstreik«. Der Mössinger Generalstreik vom 31. Januar 1933 - linker Widerstand in der schwäbischen Provinz, Stuttgart: Landeszentrale für politische Bildung Baden-Württemberg.

Friedrich-Ebert-Stiftung (Hrsg.) (1992): Textilstandort Ostdeutschland. Zukunftsperspektiven für die Textil- und Bekleidungsindustrie in den neuen Bundesländern. Tagung der Friedrich-Ebert-Stiftung am 16. Oktober 1992 in Cottbus/Brandenburg, Wirtschaftspolitische Diskurse 39 (Verfasser des Tagungsberichts: Heinrich Beyer).

Fröbel, Folker/Heinrichs, Jürgen/Kreye, Otto (1977): Die neue internationale Arbeitsteilung. Strukturell Arbeitslosigkeit in den Industrieländern und die Industrialisierung der Entwicklungsländer, Reinbek bei Hamburg: Rowohlt.

Gamillscheg, Franz (1966): Die Differenzierung nach der Gewerkschaftszugehörigkeit, Schriften zum Sozial- und Arbeitsrecht, Band 1, Berlin: Duncker \& Humblot.

Gehl, Anna (2017): Mütter und Arbeiterinnen im NS,, https://gafprojekt.hypothe ses.org/458 (Abruf am 8.1.2021).

Georg-von-Vollmar-Akademie (Hrsg.) (1971): Der Kochel-Brief II, Kochel am See.

Gertschen, Alex (2013): Klassenfeinde - Branchenpartner? Unternehmer und Gewerkschaft der westdeutschen Textilindustrie vor der Herausforderung der Internationalisierung, 1949-1979, Baden-Baden: Nomos.

Gesamtmetall (Hrsg.) (2015): 125 Jahre Gesamtmetall: Der lange Weg zur Tarifpartnerschaft, Band 2: 1990-2015, Köln: Kölner Universitätsverlag.

Gesamtmetall (2015): Tarifarchiv. Darstellung der wichtigsten Kenndaten der Tarifrunden ab 1990, www.gesamtmetall.de/sites/default/files/downloads/bro schuere_-_tarifentgelte_1990-2015.pdf (Abruf am 17.2.2021).

Gesamttextil (1996): Bündnis für Beschäftigung und Ausbildung, in: Textilbericht, Nr. 2 vom 4.4.1996. 
Gleixner, Wolfgang (1980): Die Koordinierung der Tarifpolitik durch die Bundesvereinigung der Deutschen Arbeitgeberverbände (BDA), Trier (unveröffentlichte Diplomarbeit).

Grebing, Helga (1990): Gewerkschaften: Bewegung oder Dienstleistungsorganisation - 1955 bis 1965, in: Hemmer, Hans-Otto/Schmitz, Kurt Thomas (Hrsg.): Geschichte der Gewerkschaften in der Bundesrepublik Deutschland. Von den Anfängen bis heute, Köln: Bund-Verlag, S. 149-182.

Greef, Samuel (2014): Gewerkschaften im Spiegel von Zahlen, Daten und Fakten, in: Schroeder, Wolfgang (Hrsg.): Handbuch der Gewerkschaften in Deutschland, Wiesbaden: Springer Fachmedien, S. 659-755.

Grömling, Micheal/Matthes, Jürgen (2003): Globalisierung und Strukturwandel der deutschen Textil- und Bekleidungsindustrie, IW-Analyse Nr. 1, Köln: Institut der deutschen Wirtschaft.

GTB = Gewerkschaft Textil-Bekleidung (Hrsg.) (o. J.): Die sozialen Auswirkungen der Textilindustrie. Eine Dokumentation der Gewerkschaft Textil-Bekleidung, Düsseldorf, Zentralbibliothek der IG Metall.

GTB = Gewerkschaft Textil-Bekleidung (Hrsg.) (1965): Tarifreport, Nr. 5 vom 29.3.1965, AdsD 5/GTBA410294.

GTB = Gewerkschaft Textil-Bekleidung (Hrsg.) (1965): Tarifreport Nr. 9 vom 10.05.1965, AdsD 5/GTBA410294.

GTB = Gewerkschaft Textil-Bekleidung (Hrsg.) (1991): textil-bekleidung. 100 Jahre GTB. Sonderausgabe zum Jubiläum der Gewerkschaft Textil-Bekleidung, Düsseldorf.

Guinebault, Matthieu (2018): Europas Textilimporte sinken im 1. Halbjahr, https:// de.fashionnetwork.com/news/Europas-textilimporte-sinken-im-1-halb jahr,1013946.html (Abruf am 13.2.2021).

Handelsblatt (1965): Gespaltenes Arbeitgeberlager, in: Ausgabe vom 10.3.1965.

Handelsblatt (2000): Tarifpartner einigen sich in der Textilindustrie, in: Ausgabe vom 25.9.2000.

Hardt, Wilhelm (1975): Die Textilindustrie im strukturellen Wandel, in: Vorstand der Girmes-Werke AG/Präsidium der Industrie- und Handelskammer zu Krefeld (Hrsg.): Für Erich Selbach. Zum 70. Geburtstag. 27. Juli 1975, Krefeld: Scherpe, S. 122-136.

Hassel, Anke (2003): Organisation. Struktur und Entwicklung, in: Schroeder, Wolfgang/Weßels, Bernhard: Die Gewerkschaften in Politik und Gesellschaft der Bundesrepublik Deutschland, Wiesbaden: Westdeutscher Verlag, S. 102121.

Holland, Judith (2019): Gewerkschaftliche Geschlechterpolitik. Ein deutsch-französischer Vergleich, Baden-Baden: Nomos. 
Hörath, Julia/Mielke, Siegfried (2008): Else Niewiera, in: Mielke, Siegfried (Hrsg.): Gewerkschafterinnen im NS-Staat. Verfolgung, Widerstand, Emigration, Essen: Klartext Verlag.

Horné, Alfred (1964): Sozialpolitischer Kommentar, gesendet im Bayerischen Rundfunk am 20. Januar 1964 um 18:30 Uhr, Abschrift, AdsD 5/GTBA410294.

Hübsch, Carl (1905): Der Crimmitschauer Kampf um den Zehnstundentag, Berlin: Verlag von Carl Hübsch.

Hueck, Alfred (1951): Die Bedeutung des Art. 3 des Bonner Grundgesetzes für die Lohn- und Arbeitsbedingungen der Frauen. Rechtsgutachten erstattet für die Bundesvereinigung der Deutschen Arbeitgeberverbände, Köln.

Hüren, Winfried (1994): Auch eine Frage der Ehre, in: textil-bekleidung, Ausgabe 6/1994, S. 10.

Hüser, Karl (1978): Mit Gott für unser Recht. Ein Beitrag zur Geschichte der Gewerkschaftsbewegung im Münsterland, hrsg. von der GTB-Verwaltungsstelle Emsdetten-Borghorst.

IAB = Institut für Arbeitsmarkt- und Berufsforschung (1984): Chronik der Arbeitsmarktpolitik: Vorruhestandsgesetz (VRG), http://doku.iab.de/chro nik/31/1984_05_01_31_vorr.pdf (Abruf am 13.2.2021).

ILO Vertretung in Deutschland (o. J.): Die ILO in Deutschland, www.ilo.org/ber lin/wir-uber-uns/lang--de/index.htm (Abruf am 13.2.2021).

IG Metall (Hrsg.) (2000): Textil/Bekleidung. Tarifrunde 2000. Eine Runde für uns, Frankfurt am Main.

IG Metall Bayern (Hrsg.) (2017): Vom Wiederaufbau zur Arbeit 4.o. IG Metall Bayern: 70 Jahre Fortschritt durch Tarifpolitik, Hamburg: VSA-Verlag.

IVGT = Industrieverband Veredlung - Garne - Gewebe - Technische Textilien e. V. (o. J): Das Netzwerk für die Textilindustrie. Technische Textilien, https://www. ivgt.de/fileadmin/PDF/Bericht_Deutsch_2019_kurz_FINAL.pdf (Abruf am 2.2.2021).

Jahn, Wolfgang/Jahn, Ingrid (1983): Arbeiter in der Provinz. Aspekte zur Bramscher Arbeiterbewegung von 1872-1946, Quakenbrück: Theodor Thoben.

Jünke, Christoph (2014): Das dritte Leben des Viktor Agartz, in: Globkult Magazin, 6. Dezember 2014, www.globkult.de/geschichte/personen/973-das-dritte-le ben-des-viktor-agartz (Abruf am 27.1.2021).

Keller, Berthold (1978): Frau Müller und das Hemd aus Ceylon, in: textil-bekleidung, Ausgabe 9/1978, S. 3 f.

Keller, Berthold (1982): Herausforderungen der 8oer Jahre für die Gewerkschaft Textil-Bekleidung, in: Gewerkschaftliche Monatshefte, Ausgabe 1/1982, S. 40-50.

Keller, Berthold (1982): Schwierige Zeiten gemeinsam meistern, in: textil-bekleidung, Ausgabe 1/1982, S. 7.

Keller, Berthold (1986): Unser Einsatz hat sich gelohnt, in: textil-bekleidung, Ausgabe $9 / 1986$, S. $6 \mathrm{f}$. 
Keller, Berthold (1990): Auf uns wartet noch viel Arbeit, in: textil-bekleidung, Ausgabe $1 / 1990$, S. 6.

Kittner, Michael (2005): Arbeitskampf. Geschichte - Recht - Gegenwart, München: C. H. Beck.

Kluncker, Heinz (1999): Der Gedanke der Einheitsgewerkschaft. Rede anlässlich der 50-Jahr-Feier der Gewerkschaft Öffentliche Dienste, Transport und Verkehr am 29. Januar 1999, www.verdi.de/ueber-uns/idee-tradition/ein heitsgewerkschaft/++co++4703f2bc-ec8e-11e3-a35a-5254008a33 df (Abruf am 18.1.2021).

Krämer, Susanne (1995): Viktor Agartz: Vom Cheftheoretiker zur »Persona non grata«, in: Gewerkschaftliche Monatshefte, Ausgabe 5/1995, S. 310-316.

Krippendorf, Walter/Holst, Gregor/Richter, Ursula (2009): Branchenanalyse Textilindustrie. Untersuchungen zur Situation und Entwicklung der Branchen »Textilgewerbe«(WZ 17). Projektbericht an die Hans-Böckler-Stiftung, Berlin: IMU-Institut.

Kuhn, Annette (Hrsg.) (1994): Frauenleben im NS-Alltag. Bonner Studien zur Frauengeschichte, Pfaffenweiler: Centaurus.

Linde, Gerda (1984): Wachsender Druck auf die erwerbstätige Frau, in: textil-bekleidung, Ausgabe 3/1984, S. 14.

Linde, Gerda (1985): Frauen sind die Verlierer der Wende-Politik, in: textil-bekleidung, Ausgabe 7-8/1985, S. 18.

Lindner, Oda (1978): Wann sind wir aus dem Schneider? Frauenerwerbstätigkeit und Gewerkschaft Textil und Bekleidung, Berlin: Verlag Die Arbeitswelt.

Lindner, Stephan H. (2001): Den Faden verloren, München: C. H. Beck.

Loderer, Eugen (1982): Für eine arbeitsorientierte Wirtschaftspolitik, in: Gewerkschaftliche Monatshefte, Ausgabe 1/1982, S. 16-22.

Lomba, E. R. (1959): Von Leuten, die im Trüben fischen, in: Allgemeine Sonntagszeitung, Nr. 16 vom 19.4.1959.

Lösch, Hans Peter (1969): Die Textilkonjunktur im allgemeinen Wirtschaftsablauf, Forschungsberichte des Landes Nordrhein-Westfalen, Nr. 2020, Wiesbaden: Springer Fachmedien.

Maunz, Dürig (2018): Grundgesetz-Kommentar, 84. Auflage, München: C. H. Beck.

Mayr, Hans (1984): Der Kampf um die 35-Stunden-Woche. Erfahrungen und Schlussfolgerungen aus der Tarifbewegung 1984, in: Gewerkschaftliche Monatshefte, Ausgabe 11/1984, S. 661-671.

Meißner, Martina (2013): Erste Mutterschutzbestimmungen im Dt. Reich, Erlass (am 07.07.1878), WDR ZeitZeichen, www1.wdr.de/mediathek/audio/zeitzei chen/audio-erste-mutterschutzbestimmungen-im-dt-reich-erlass-am--100. html (Abruf am 13.2.2021). 
Menschik, Jutta (1974): Gleichberechtigung oder Emanzipation? Die Frau im Erwerbsleben der Bundesrepublik, 4., überarbeitete Auflage, Frankfurt am Main: Fischer.

Morgenroth, Christine (1996): Die engagierte Frau. Frauen und Interessenorganisationen, Münster: Verlag Westfälisches Dampfboot.

Müller-Jentsch, Walther (1997): Soziologie der industriellen Beziehungen, Frankfurt am Main/New York: Campus.

Naffin, Beate (2002): Kritische Akademie Inzell. »Hier werden keine Revolutionäre gemacht«, Düsseldorf: Stiftung zur Förderung von Bildung und Erholung der Arbeitnehmer der Miederindustrie.

Netzwerk Industrie.Kultur.Ost (o. J.): Die Textilindustrie. Aufstieg \& Fall einer Wirtschaftsmacht, www.industrie-kultur-ost.de/streiflichter/die-textilindu strie-aufstieg-und-fall-einer-wirtschaftsmacht (Abruf am 16.1.2021).

Notz, Gisela (2003): Frauen in der Mannschaft. Sozialdemokratinnen im Parlamentarischen Rat und im Deutschen Bundestag 1948/49-1957, Bonn: J. H. W. Dietz Nachf.

Pelke, Else (1954): Zwischen Arbeit und Familie: Die berufstätige Frau, in: textilbekleidung, Ausgaben vom 14.7.1954 und 1.8.1954.

Pfleger-Edel, U. (1976): Der Fall Glöggler hat Textilindustrie psychologischen Schock versetzt, in: Handelsblatt vom 26.3.1976.

Pinl, Claudia (1977): Das Arbeitnehmerpatriarchat. Die Frauenpolitik der Gewerkschaften, Köln: Kiepenheuer \& Witsch.

Preuß, Olaf (2007): Eine Kiste erobert die Welt. Der Siegeszug einer einfachen Erfindung, Hamburg: Murmann.

Resch, Marianne/Rummel, Martina (1986): Von allem die Hälfte. Frauen im Beruf, Köln: Bund-Verlag.

Ricardo, David (1817): The Principles of Political Economy and Taxation, London: John Murray.

Richter, Eberhard (1975): Vorurteile gegenüber Frauen - Ursachen und Wirkungen, Vortrag, in: Weber, Maria (Hrsg.): Probleme der Frauen - Probleme der Gesellschaft. Arbeitschancen, Lohngleichheit, Vorurteile. Protokoll der Arbeitstagung des DGB am 6.-7. November 1975 in Leverkusen, Köln/Frankfurt am Main: Europäische Verlagsanstalt, S. 47-58.

Riebl, Reinhold/Kuhn, Axel (1979): Die Anfänge der Gewerkschaften in Esslingen bis 1878, hrsg. vom Deutschen Gewerkschaftsbund, Kreis Esslingen.

Ringelstein, Ernst (1985): Klartext geredet, in: textil-bekleidung, Ausgabe 1/1985, S. 14.

Rölke, Kirsten/Wilke, Christiane/Kopel, Mechthild (2006): Gleich gestellt: doppelt stark. Chancengleichheit in Unternehmen, Hamburg: VSA-Verlag.

Rüther, Ernst (1968): Textilgewerkschaft zwischen Reformismus und Radikalismus, in: Gesellschaftspolitische Kommentare, Nr. 5 vom 1.3.1968, S. 59 f. 
Schambach-Hardtke, Lydia (2005): Gender und Gewerkschaften. Der Kampf von Frauen um politische Partizipation im organisatorischen Wandel, Opladen: Barbara Budrich.

Schlemmer, Thomas/Woller, Hans (Hrsg.) 2004: Politik und Kultur im föderativen Staat, München: Oldenbourg.

Schneider, Michael (1989): Kleine Geschichte der Gewerkschaften, Bonn: J. H. W. Dietz Nachf.

Schmitz, Kurt Thomas (2020): Die IG Metall nach dem Boom. Herausforderungen und strategische Reaktionen, Bonn: J. H. W. Dietz Nachf.

Schönhoven, Klaus (1980): Wegbereiter der sozialen Demokratie? Zur Bedeutung des Stinnes-Legien-Abkommen vom 15. November 1918, in: Führer, Karl Christian/Schildt, Axel/Tenfelde, Klaus (Hrsg): Revolution und Arbeiterbewegung in Deutschland. 1918-1920, Essen: Klartext Verlag S. 61-79.

Schönhoven, Klaus (2014): Geschichte der deutschen Gewerkschaften: Phasen und Probleme, in: Schroeder, Wolfgang (Hrsg): Handbuch Gewerkschaften in Deutschland, 2. Aufl., Wiesbaden: Springer Fachmedien, S. 59-84.

Schrader, Karl (1931): Der Deutsche Textilarbeiter-Verband, in: Ludwig Heyde (Hrsg.): Internationales Handwörterbuch des Gewerkschaftswesens, Berlin: Verlag Neue Gesellschaft, S. 75-78 (Nachdruck: Archiv für Sozialgeschichte, Band 21, Bonn: J. H. W. Dietz Nachf.).

Schroeder, Wolfgang (2000): Das Modell Deutschland auf dem Prüfstand. Zur Entwicklung der industriellen Beziehungen in Ostdeutschland, Wiesbaden: Westdeutscher Verlag.

Schroeder, Wolfgang (Hrsg.) (2014): Handbuch Gewerkschaften, Wiesbaden: Springer VS.

Schroeder, Wolfgang/Keudel, Dorothea (2008): Strategische Akteure in drei Welten. Die deutschen Gewerkschaften im Spiegel der neueren Forschung, Düsseldorf: Hans-Böckler-Stiftung.

Schroeder, Wolfgang/Weßels, Bernhard (Hrsg.) (2010): Die Arbeitgeber- und Wirtschaftsverbände in Deutschland, Wiesbaden: VS Verlag für Sozialwissenschaften.

Schumacher, Anja (o. J.): $\$ 9$ Kündigung im Arbeitskampf / 5. Sonderfall: Lösende Aussperrung, www.haufe.de/recht/deutsches-anwalt-office-premium/9kuendigung-im-arbeitskampf-5-sonderfall-loesende-aussperrung_idesk_ PI17574_HI10974665.html (Abruf am 5.2.2021).

Schumacher, Hermann (1987): Mehr Kaufkraft - mehr Schutz, in: textil-bekleidung, Ausgabe 4/1987, S. 7.

Schütz, Uwe (2012): Vor 50 Jahren: Rundfunkansprache von Bundeswirtschaftsminister Ludwig Erhard, www.aref.de/kalenderblatt/2012/12_mass-halten_ ludwig-erhard_rundfunkansprache_1962.php (Abruf am 5.2.2021). 
Schwarzer, Alice (1973): Frauenarbeit - Frauenbefreiung, Frankfurt am Main: Suhrkamp.

Schwarzer, Alice (1975): Der kleine Unterschied und seine großen Folgen. Frauen über sich. Beginn einer Befreiung, Frankfurt am Main: Suhrkamp.

Silvia, Stephen John (1990): Jobs, trade and unions. The politics of employment and protectionism in West Germany and America, New York: ProQuest Dissertations \& Theses.

Sinn, Gerlinde/Sinn, Hans-Werner (1992): Kaltstart. Volkswirtschaftliche Aspekte der deutschen Vereinigung, 2. Aufl., Tübingen: J. C. B. Mohr (Paul Siebeck).

Spode, Hasso/Volkmann, Heinrich/Morsch, Günter/Hudemann, Rainer (1992): Statistik der Arbeitskämpfe in Deutschland, Quellen und Forschungen zur historischen Statistik von Deutschland, Bd. 15, Sankt Katharinen: Scripta Mercaturae.

Statistisches Bundesamt (1952): Wirtschaft und Statistik, 4. Jahrgang, Heft 8.

Statistisches Bundesamt (1970): Reihe 15.

Statistisches Bundesamt (1990): Fachserie 16, Reihe 2.1.

Statistisches Bundesamt (Destatis) (o. J.): Einkommens- und Verbrauchsstichprobe (EVS; verschiedene Jahrgänge).

Statistisches Bundesamt (Destatis) (2008): Fachserie 4, Reihe 4.1.1, Beschäftigung und Umsatz der Betriebe des Verarbeitenden Gewerbes sowie des Bergbaus und der Gewinnung von Steinen und Erden.

Statistisches Bundesamt (Destatis) (2017): Verdiensterhebung im Produzierenden Gewerbe, Handel, Kredit- und Versicherungsgewerbe ab 1950, Zeitreihe 1623148 (Archiv-CD).

Statistisches Bundesamt (Destatis) (2018): Volkswirtschaftliche Gesamtrechnungen. Inlandsproduktberechnungen ab 1970.

Statistisches Bundesamt (Destatis) (2019): Volkswirtschaftliche Gesamtrechnungen. Inlandsproduktberechnung. Lange Reihen ab 1970, Fachserie 18, Reihe 1.5 .

Statistisches Bundesamt (Destatis) (2020): Verdiensterhebung im Produzierenden Gewerbe 1950-1995, Zeitreihen 2155 und 1623 (Archiv-CD).

Statistisches Bundesamt (Destatis) (2021): Preise. Verbraucherpreisindizes für Deutschland. Lange Reihen $a b$ 1948, www.destatis.de/DE/Themen/Wirtschaft/Preise/Verbraucherpreisindex/Publikationen/Downloads-Verbrau cherpreise/verbraucherpreisindex-lange-reihen-pdf-5611103.pdf (Abruf am 17.2.2021).

Statistisches Bundesamt (Destatis) (2021): Volkswirtschaftliche Gesamtrechnungen. Bruttoinlandsprodukt, Bruttonationaleinkommen, Volkseinkommen. Lange Reihen ab 1925, www.destatis.de/DE/Themen/Wirtschaft/Volkswirtschaftliche-Gesamtrechnungen-Inlandsprodukt/Tabellen/inlandsproduktvolkseinkommen1925-pdf.pdf (Abruf am 16.1.2021). 
Stender, Wolfgang (1990): Konflikt um jeden Preis?, in: textil-bekleidung, Ausgabe $5 / 1990$, S. 3 .

Stender, Wolfgang (1992): Das Tor zur Zukunft geöffnet, in: textil-bekleidung, Ausgabe 7-8/1992, S. 6 ff.

Stender, Wolfgang (1993): Hart errungene Kompromisse, in: textil-bekleidung, Ausgabe 9/1993, S. 6 f. und 10.

Stender, Wolfgang (2011): Saga, Eigenverlag.

Stiftung zur Förderung von Bildung und Erholung der Arbeitnehmer der Miederindustrie (Hrsg.) (1970): Denkschrift. Die Kritische Akademie. Ein Modell, Düsseldorf.

Streeck, Wolfgang (1978): Politischer Wandel und organisatorische Reformen, in: Gewerkschaftliche Monatshefte, Ausgabe 10/1978, S. 589-601.

Stuttgarter Zeitung (1965): Stoß vom Trittbrett, in: Ausgabe vom 31.3.1965.

Verein zur Weiterbildung der Arbeitnehmerinnen und Arbeitnehmer in der Textilund Bekleidungsindustrie (Hrsg.) (1998): Die GTB 1990-1998, Düsseldorf.

Vietheer, Heinz (1961): Koordination und Konzentration im gewerkschaftlichen Bereich, in: Gewerkschaftliche Monatshefte, Ausgabe 3/1961, S. 162-165.

Vietheer, Heinz (1971): Zur Diskussion um die DGB-Reform - groß oder klein - ist das ein Problem?, in: Gewerkschaftliche Monatshefte, Ausgabe 4/1971, S. 202205.

Vogel, Otto A. H. (1950): Rede auf der Anti-Mitbestimmungs-Kundgebung »Der deutsche Unternehmer. Leistung und Verpflichtung« des BDI am 8. November 1950 in Köln, BWA No2 53.

Wassermann, Wolfram (1985): Arbeitsgestaltung als Gegenstand gewerkschaftlicher Politik. Zur Soziologie der Arbeitsgestaltung am Beispiel der Textil- und Bekleidungsindustrie, Bonn: Verlag Neue Gesellschaft.

Weber, Maria (1972): Programmatische Forderungen des DGB zum Jahr der Arbeitnehmerin, in: Gewerkschaftliche Monatshefte, Ausgabe 11/1972, S. 673-687.

Weber, Maria (Hrsg.) (1976): Probleme der Frauen - Probleme der Gesellschaft. Arbeitschancen, Lohngleichheit, Vorurteile. Protokoll der Arbeitstagung des DGB am 6.-7. November 1975 in Leverkusen, Köln/Frankfurt am Main: Europäische Verlagsanstalt.

Wedde, Peter (Hrsg.) (2009): Arbeitsrecht. Kompaktkommentar zum Individualarbeitsrecht mit kollektiven Bezügen, Köln: Bund-Verlag.

Weidenhausen, Evelyn Mejrem (2010): Globalisierungsprozesse in der Textilwirtschaft, insbesondere in der ökologisch ausgerichteten Branche, Reutlingen (Dissertation).

Westdeutsche Allgemeine Zeitung (1965): Organisierten steht Extrarecht zu, in: Ausgabe vom 1.4.1965.

Wikipedia-Artikel »Frauenanteil im Deutschen Bundestag seit 1949«, Tabelle 1, https://de.wikipedia.org/wiki/Frauenanteil_im_Deutschen_Bundestag_ 
seit_1949\#Tabelle_1:_Frauenanteil_des_Deutschen_Bundestags_seit_1949_ (gesamt) (Abruf am 11.2.2021).

Wikipedia-Artikel »Ruhrbergbau«, Abschnitt »Geschichte«, https://de.wikipedia. org/wiki/Ruhrbergbau\#Geschichte (Abruf am 16.1.2021).

Wikipedia-Artikel »Technische Textilie«, Abschnitt »Historische Entwicklung«, https://de.wikipedia.org/wiki/Technische_Textilie\#Historische_Entwicklung (Abruf am 15.1.2021).

Wroblewski, Angela/Leitner, Andrea (2005): Lernen von den Besten. Interdependenzen von Frauenerwerbsbeteiligung und Geburtenzahlen im Ländervergleich, Düsseldorf: Hans-Böckler-Stiftung.

www.gewerkschaftsgeschichte.de (o. J.): Interne Debatte - Uneins über die Rolle des DGB, https://www.gewerkschaftsgeschichte.de/rolle-des-dgb-umstritten.html (Abruf am 28.1.2021).

Zonenausschuß der CDU für die britische Zone (1947): Ahlener Programm, Ahlen/Westfalen, 3. Februar 1947, www.kas.de/c/document_library/get_file?uu $\mathrm{id}=76 \mathrm{a} 77614-6803-0750-\mathrm{c} 7 \mathrm{a} 7-5 \mathrm{~d} 3 \mathrm{ff} 7 \mathrm{c} 46206$ \&groupId=252038 (Abruf am 27.1.2021). 